\title{
Haemoptysis as the sole presenting symptom of dissection of the aorta
}

\author{
A SHARON GUIDETTI, A PIK, A PEER, S SHIKIAR, D BEN-YAAKOV
}

From the Departments of Internal Medicine D and Radiology, Assaf Harofeh Medical Center, Zerifin, and Tel Aviv University Sackler School of Medicine, Tel Aviv, Israel

ABSTRACT A 75 year old man who had had haemoptysis for 24 hours was found to have his left lower lobe compressed by a dissection of the aorta, which was otherwise symptomless.

Haemoptysis is a rare complication of aortic dissection, and recurrent haemoptysis as the sole initial symptom is most unusual. We report such a case.

\section{Case report}

A 75 year old man was admitted to our department because of recurrent haemoptysis of 24 hours' duration. His medical history included hypertension and mild diabetes mellitus of five years' duration. He had been admitted to hospital two years earlier because of recurrent transient ischaemic attacks.

The patient was symptomless and on physical examination was generally well, without dyspnoea or chest pain. The pulse was regular at 100 beats/min and the blood pressure was $160 /$ $110 \mathrm{~mm} \mathrm{Hg}$. No murmurs were heard over the heart; breath sounds were diminished over the lower part of the left lung. The femoral arteries were palpated but peripheral pulses were absent.

The pertinent laboratory data included packed cell volume $(0.51)$ and prothrombin time, platelet count, and creatinine concentration, which were all within the normal range. No acid fast bacilli were identified in sputum.

An initial chest radiograph showed a tortuous and enlarged aorta with a questionable double contour at the left edge of the aortic arch. Computed tomography showed an enlarged aneurysmal aorta with an intimal flap (fig 1). Irregular crowding of the lower left lobe due to compression by the aneurysm was also seen (fig 2). Subsequent angiography confirmed dissection of the descending aorta. At bronchoscopy the only abnormal finding was a clot in the left lower lobe bronchus.

The patient refused surgical intervention. Paraparesis developed and he died two weeks later in uraemic coma. Postmortem examination showed dissection of the descending aorta beyond the origin of the left subclavian artery, adhesions of the aneurysm along the left lung, and an infected area in the lung adjacent to the aneurysm.

Address for reprint requests: Dr A Pik, Department of Internal Medicine D, Assaf Harofeh Medical Center, 70300 Zerifin, Israel.

Accepted 6 January 1989

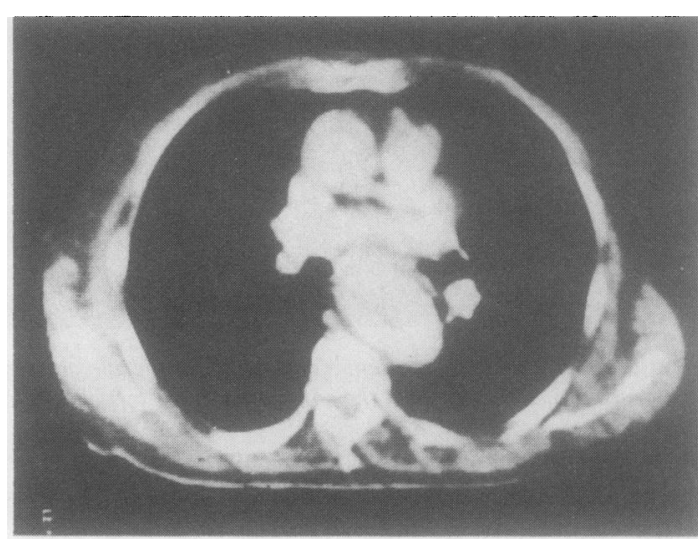

Fig 1 Computed tomography scan showing an aneurysmal enlargement of the descending aorta with an intimal flap.

\section{Discussion}

The characteristic clinical picture of dissection of the aorta is dominated in more than $90 \%$ of cases by severe and unremitting pain.' Painless dissection has been described, ${ }^{23} \times$ but in the absence of pain the diagnosis is frequently $\frac{\sigma}{3}$ overlooked. Bleeding due to oozing or rupture from adissecting aneurysm into the pleural cavity is a knowno

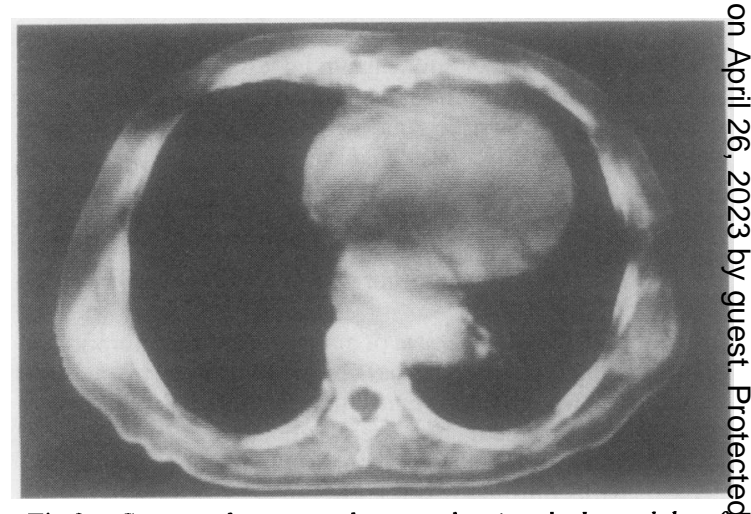

Fig 2 Computed tomography scan showing the lower lobe of the left lung compressed by the aneurysm. 
complication. Haemoptysis, on the other hand, has been described only rarely.

The haemoptysis in our case may have been due to pressure from the aneurysm on the lung, causing collapse and infection, or alternatively erosion of a small vessel of the compressed lung by the aneurysm.

No communication between the aneurysm and lung was found at necropsy, though the lung adjacent to the aneurysm was infected. We presume therefore that the haemoptysis was due to compression of the lower lobe of the left lung by the aneurysm.

Recurrent haemoptysis as the sole presenting symptom of dissecting aneurysm has not been previously described. In a review of 130 cases of haemoptysis this aetiology was not mentioned. ${ }^{5}$ Despite its rarity as a cause, we suggest that dissecting aneurysm should be included in the differential diagnosis of haemoptysis of obscure aetiology.

\section{References}

1 Slater EE, Desanctis RW. Dissection of the aorta. Med Clin North Am 1979;63:141-54.

2 Cohen S, Littmann D. Painless dissecting aneurysm of the aorta. $N$ Engl J Med 1964;271:143-5.

3 Wesolowski SA, Schaefer HC, Domingo RT, Greenfield H, Lyons HP, Sawyer PN. A new radiographic sign of aortic dissection. Surgery 1962;51:699-704.

4 Barbasah G, Salomon A, Reider I, Grosswasser B, Bidne A, Almog H. Aortic dissection presenting as haemoptysis. Diagnostic confirmation of dissection and leak by computerised tomography. Heart Lung 1983;12:633-5.

5 Grief J, Cohen R, Topensky M. Diagnosis of haemoptysis. Harefuah 1985;108:6-8. 